\title{
Temperature Distribution to Fluid Transport in a Vertical Porous Tube under the Action of Peristalsis
}

\author{
V. Radhakrishna Murthy, P. Sudam Sekhar
}

\begin{abstract}
Peristaltic transport of a Newtonian fluid, with heat transfer, in a vertical porous axisymmetric tube under long wave length approximation is considered. Closed form solution is obtained as an asymptotic expansion in terms of porosity and free convection parameters. Expressions for temperature, coefficient of heat transfer and pressure -flow relationship at the boundary wall of the tube are derived. It is observed that pressure drop increases as amplitude ratio increases. Further, it has been observed that for some specific values of otherparameters under consideration the mean flux significantly increases by about 8 to 10 percent as Grashof number increasesfrom 1 to 2.
\end{abstract}

Key words- Peristalsis, heat transfer, porosity, pressure drop, mean flux.

\section{INTRODUCTION}

Peristalsis is a mechanism for fluid transport which is achieved by the passage of area contraction and expansion waves along the length of the distensible tube. The peristaltic transport may be useful in processes where it is desirable to avoid using internal moving parts such as piston in the pumping process. It is known to be one of the main mechanisms for fluid transport in physiological systems, in particular, urine passage through Ureter, food mixing and chyme movement in the intestines. Based on this principle, a blood pump in dialysis is designedto prevent the transported fluid from being contaminated. Peristaltic transport of toxic liquid is used in nuclear industries so as not to contaminate outside environment. been carried out theoretically and experimentallyby various authors [1-12]. In particular, Radhakrishnamacharya[1] investigated peristaltic pumping in an axisymmetric tube under long wave length approximation using power law fluid model. Takabatake et al [2] have developed complete numerical solutions for peristaltic pumping and its efficiency in Cartesian and axisymmetric geometries. Recently, the thermo dynamical aspects of peristalsis have received attention [3-7] as it might be relevant in certain processes like oxygenation and hemodialysis.

Translocation of water in tall trees is a phenomenon which is not well understood by scientists for well over centuries. It is speculated that peristalsis might be involved in this process sincediameters of trunks of the trees are found to vary with time. Hence some authors [10-11] have investigated the peristalsis phenomena particularly in the case of transport of water in trees.

Revised Manuscript Received on December 15, 2019

V. Radhakrishna Murthy, Professor, Division of Mathematics, Vignan's Foundation for Science, Technology and Research (Deemed to be University), Guntur, India. E-mail: drvrm_sh@ac.in

P. Sudam Sekhar, Associate Professor, Division of Mathematics, Vignan's Foundation for Science, Technology and Research (Deemed to be University), Guntur, India. E-mail: drpss_sh@ vignan.ac.in
In view of its importance,studies of peristaltic transport have

Further, it is observed that flow of water takes place through the porous matrix of the tree.Keeping in view of the above observations, in this paper an attempt has been made, to study heat transfer for the Newtonian fluid flow under the action of peristalsis in a vertical axisymmetric porous tube. Assuming long wave length approximation analytical expression for temperature, pressure drop and heat transfer coefficient have been derived and closed form solutions in terms of porosity $\left(\sigma^{2}\right)$ and free convection $\left(G_{m}\right)$ parameters have been obtained using a perturbation solution. From the analysis it has been observed that for fixed values of other parameters a small change in free convection leads to significant increase in mean flux.

\section{MATHEMATICAL FORMULATION}

The governing flow of a Newtonian, incompressible fluid through an axisymmetric vertical tube, filled with porous material is considered. Peristaltic waves of very large wave length are assumed to travel down the wall of the tube. In this problem the cylindrical polar coordinate $(\mathrm{X}, \mathrm{R})$ is chosen where $\mathrm{X}$ represents axial coordinate and $\mathrm{R}$ is radial coordinate.

The simplified, zeroth order equations, under long wave approximation, governing the flow $[8,9,10]$ are

$$
0=-\frac{\partial p}{\partial X}+\frac{\mu}{R} \frac{\partial}{\partial R}\left(R \frac{\partial W}{\partial R}\right)-\frac{\mu}{k_{0}} W+\rho g \beta\left(T-T_{0}\right)
$$

$$
\begin{aligned}
& 0=\frac{\partial W}{\partial X}+\frac{U}{R}+\frac{\partial U}{\partial R} \\
& 0=\frac{K}{R} \frac{\partial}{\partial R}\left(R \frac{\partial T}{\partial R}\right)+\mu\left(\frac{\partial W}{\partial R}\right)^{2}+\frac{\mu}{k_{0}} W^{2}
\end{aligned}
$$

The equation of the tube wall is given by

$$
H(X, t)=a+b \operatorname{Sin}\left(\frac{2 \pi}{\lambda}\right)(X-c t)
$$

Where the fluid velocity components are $\mathrm{W}$ and $\mathrm{U}$ in the direction $\mathrm{X}$ and $\mathrm{R}$ respectively, $\mathrm{T}$ is the fluid temperature, $T_{0}$ is the boundary temperature, ' $p$ ' is the pressure , ' $\rho$ ' is the density, the coefficient of expansion is $\beta$,' $\mathrm{k}$ 'stands for thermal conductivity of the fluid, ' $\mu$ ' is viscosity coefficient , ' $\mathrm{g}$ ' is the acceleration due to gravity, 'c' is the phase speed of the wave, ' $k_{0}$ 'is the permeability of the medium, 'a' is the mean radius of the tube, ' $b$ ' is the amplitude and the wave length is ${ }^{\prime} \lambda^{\prime}$.The assumed boundary conditions for the problem under considerations are

$\mathrm{T}=T_{0}$ and $\mathrm{W}=0$ at $\mathrm{R}= \pm \mathrm{H}$ (5) 
Relative to the laboratory frame the wave frame of reference moves with a constant speed ' $c$ '. In the wave frame the measurement of the variables ' $x$ ' and ' $r$ 'are defined by

$\mathrm{x}=\mathrm{X}-\mathrm{ct}, \mathrm{r}=\mathrm{R}$

(6)

The corresponding velocity components of the fluid are $\mathrm{w}=\mathrm{W}-\mathrm{c}, \mathrm{u}=\mathrm{U}$

The governing system of equations of the fluid flow in the wave frame of reference are written as

$0=-\frac{\partial p}{\partial x}+\frac{\mu}{r} \frac{\partial}{\partial r}\left(r \frac{\partial w}{\partial r}\right)-\frac{\mu}{k_{0}}(w+c)+\rho g \beta\left(T-T_{0}\right)$

$0=\frac{\partial w}{\partial x}+\frac{u}{r}+\frac{\partial u}{\partial r}$
$0=\frac{k}{r} \frac{\partial}{\partial r}\left(r \frac{\partial T}{\partial r}\right)+\mu\left(\frac{\partial w}{\partial r}\right)^{2}+\frac{\mu}{k_{0}}(w+c)^{2}$

The boundary conditions are $\mathrm{w}=-\mathrm{c}$ and $\mathrm{T}=\mathrm{T}_{0}$ at $r=a+b \sin \left(\frac{2 \pi}{\lambda}\right) x$

The non-dimensional quantities can be introducedas

$$
F=\left(F_{00}+G_{m} F_{01}+\ldots \ldots\right)+\sigma^{2}\left(F_{10}+\ldots\right.
$$

Where, the flow variable is denoted as F.

By solving the resulting equations which are obtained by using (18) in equation (13), (15) and (16), the solutions for velocity component ' $w$ ' and temperature ' $\theta$ ' can be obtained under the suitable boundary conditions, However, the expressions for velocity component $\mathrm{w}$ is not presented here and the expressions for temperature $\theta$ is $\theta=\left(\theta_{00}+G_{m} \theta_{01}+\ldots \ldots\right)+\sigma^{2}\left(\theta_{10}+\ldots ..\right)+$

Where

$$
\begin{aligned}
& \theta_{00}=\frac{-1}{64} E_{m} \alpha^{2}\left(r^{4}-\eta^{4}\right) \theta_{01}=\frac{-1}{32} E_{m}\left[\alpha \tau\left(r^{4}-\eta^{4}\right)+\frac{E_{m}}{768}\left(r^{8}-12 r^{4} \eta^{4}+11 \eta^{8}\right)\right] \\
& \theta_{10}=\frac{-1}{32} E_{m}\left[\alpha \gamma\left(r^{4}-\eta^{4}\right)+\frac{\alpha^{2}}{36}\left(2 r^{6}-9 r^{4} \eta^{2}+7 \eta^{6}\right)\right]-\frac{\alpha^{2}}{72}\left(2 r^{6}-9 r^{4} \eta^{2}+18 r^{2} \eta^{4}-20 \eta^{6}\right)
\end{aligned}
$$

$$
\begin{aligned}
& \alpha=\frac{8\left(\overline{Q_{00}+\pi}\right)}{\pi\left(1-2 \eta^{2}\right)} \\
& \tau=\frac{1}{\pi\left(1-2 \eta^{2}\right)}\left[32 \bar{Q}_{01}-\frac{1}{9} E_{m} \alpha^{2}\left(140 \eta^{6}-18 \eta^{4}\right)+1\right]
\end{aligned}
$$

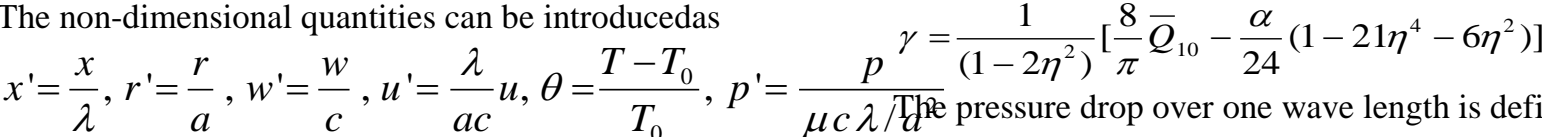

in to the equations (8) - (11), we get (after dropping the primes)

$$
0=-\frac{\partial p}{\partial x}+\frac{1}{r} \frac{\partial}{\partial r}\left(r \frac{\partial w}{\partial r}\right)-\sigma^{2}(w+1) G_{m} \theta
$$

$0=\frac{\partial w}{\partial x}+\frac{u}{r}+\frac{\partial u}{\partial r}$

$\mathrm{O}=\frac{1}{r} \frac{\partial}{\partial r}\left(r \frac{\partial \theta}{\partial r}\right)+E_{m}\left(\frac{\partial w}{\partial r}\right)^{2}+\sigma^{2} E_{m}(w+1)^{2}$

The boundary conditions for the dimensionless quantities are

$$
w=-1 \text { and } \theta=0 \text { at } r= \pm \eta(x)
$$

Where $\eta(x)=1+\varepsilon \operatorname{Sin} 2 \pi x$ $\sigma^{2}=\frac{a^{2}}{k_{0}}($ Porosity parameter $), G_{m}=\frac{g \beta T_{0} a^{3}}{v^{2}}($ Grashof number $)$ $E_{m}=\frac{\mu c^{2}}{K T_{0}}($ Ecker $\operatorname{tnumber})$ and $\varepsilon=\frac{b}{a}$ (amplituderatio $)$

\section{III.}

\section{ANALYSIS}

Equations (13) and (15) are simultaneous non-linear equations hence for arbitrary values of all the parameters it is quite impossible to get an exact solution. So, we opt perturbation method in the form of a series as
$\Delta p_{\lambda}=\int_{0}^{\lambda} \frac{\partial p}{\partial x} d x$

Substituting the relation for $\frac{\partial p}{\partial x}$ from equation (13) in (20) and using expressions for velocity (which is not presented) and temperature from equation (19), the non-dimensional pressure drop can finally be obtained as

$$
\Delta p=\frac{\Delta p_{\lambda}}{\frac{\mu c \lambda}{a^{2}}}=\left(\Delta p_{00}+G_{m} \Delta p_{01}+\ldots \ldots \ldots\right)+\sigma^{2}\left(\Delta p_{10}+\ldots \ldots . .\right)+\ldots \ldots \ldots . .
$$

Where,

$$
\begin{aligned}
& \Delta p_{00}=\frac{32 \varepsilon^{2}\left(1-\frac{\varepsilon^{2}}{16}\right)-8 \bar{Q}_{00}\left(1+\frac{3 \varepsilon^{2}}{2}\right)}{\left(1-\varepsilon^{2}\right)^{7 / 2}} \\
& \Delta p_{01}=\frac{5}{6} E_{m}\left[1+\left(\bar{Q}_{00}-\left(1+\frac{\varepsilon^{2}}{2}\right)^{2}\right)\left(\frac{\left(1+\frac{3 \varepsilon^{2}}{2}\right)}{\left(1-\varepsilon^{2}\right)^{7 / 2}}+\frac{\bar{Q}_{00}-\left(1+\frac{\varepsilon^{2}}{2}\right)}{\left(1-\varepsilon^{2}\right)^{9 / 2}}\right)-8 \bar{Q}_{01} \frac{\left(1+\frac{3 \varepsilon^{2}}{2}\right)}{\left(1-\varepsilon^{2}\right)^{7 / 2}}\right. \\
& \Delta p_{10}=-6 \bar{Q}_{10}\left[\frac{2+\varepsilon^{2}}{2\left(1-\varepsilon^{2}\right)^{5 / 2}}\right]-\frac{7}{20}\left[1+\frac{\bar{Q}_{00}-1}{\left(1-\varepsilon^{2}\right)^{9 / 2}}\right]
\end{aligned}
$$

And $\bar{Q}$ is the dimensionless mean flux.

The non-dimensional form of heat transfer coefficient $\mathrm{Z}$ on the boundary of the tube is given by $Z=\left(\frac{\partial \theta}{\partial x}+\frac{\partial \theta}{\partial r} \frac{\partial \eta}{\partial x}\right) a t r=\eta$

Which in view of (18), can be expressed as 
$Z=\left(Z_{00}+G_{m} Z_{01}+\ldots ..\right)+\sigma^{2}\left(Z_{10}+\ldots ..\right)+\ldots \ldots$.

Where

$$
Z_{00}=\frac{\partial \theta_{00}}{\partial r} \frac{\partial \eta}{\partial x}, Z_{01}=\frac{\partial \theta_{01}}{\partial r} \frac{\partial \eta}{\partial x}, Z_{10}=\frac{\partial \theta_{10}}{\partial r} \frac{\partial \eta}{\partial x}
$$

\section{RESULTS AND DISCUSSION}

Analytical expressions for temperature, pressure drop and the coefficient of heat transfer are given by the equations (19), (21) and (22) respectively. To study explicitly these quantities are numerically evaluated and the results are presented in fig: 1 -to fig: 4 and Table- 1 to Table -4 . Here the effects of various parameters on these flow variables are depicted clearly.

Figs 1 -4 show the variation of temperature vs. $\mathrm{X}$ with respect to various parameters. It is observed from Figs. $1-4$ that for a fixed values of certain parameters, temperature first increases down the tube and then decreases. This may be due to the effects of peristalsis. From figs. 1 and 2, we can see that, the temperature increases as Eckert number $\left(E_{m}\right)$ or Grashof number $\left(G_{m}\right)$ increases when other parameters are made fixed. Further, the temperature increases as $\sigma^{2}$ ore increases i.e. the temperature increases as the tube becomes more porous or peristaltic wave amplitude increases (Figs 3 and 4).

It has been observed that the mean flux, $\bar{Q}$, increases by about 8 to 10 percent as $G_{m}$ increases from 1 to 2 when some other parameters are given a fixed value during the computational process.

Heat transfer coefficient, $Z$, on the boundary of the tube, is evaluated numerically and the results are presented in tables 1- 4. $\mathrm{Z}$ increases down the tube and then decreases, in the case of temperature, which may be due to peristalsis.

From table 1 and 2, we can see that, by making some parameters fixed during the computation, there is an increase in the heat transfer coefficient as Eckert number or Grashof number increases. Tables 3 and 4 show that $\mathrm{Z}$ increase with $\varepsilon$ while it decreases with porosity [11-12].

\section{CONCLUSION}

In the above results it has been observed that temperature is increases for some specific values of differentparameters under consideration which is shown in fig- 1 to fig-4. Further it has been observed that the absolute value of the temperature significantly increases by about 8 to 10 percent as increase in 1 to 2 in Grashof number $\left(G_{m}\right)$ which is shown in table- 1 to table- 4 .

\section{TABLE -1}

Heat transfer variation with $\mathrm{E}_{\mathrm{m}}$ $\left(\mathrm{G}_{\mathrm{m}}=3, \sigma^{2}=2, \varepsilon=0.1\right)$

\begin{tabular}{|l|l|l|l|}
\hline $\mathrm{x}$ & $\mathrm{E}_{\mathrm{m}}=1$ & $\mathrm{E}_{\mathrm{m}}=3$ & $\mathrm{E}_{\mathrm{m}}=5$ \\
\hline 0.0 & 2.42945 & 22.0037 & 61.17046 \\
\hline 0.4 & 2.84512 & 25.72656 & 71.150905 \\
\hline 0.8 & 0.39168 & 3.56543 & 9.91642 \\
\hline
\end{tabular}

TABLE -2

Variation of heat transfer with respect to $\mathrm{Gm}$ $\left(\sigma^{2}=2, \varepsilon=0.1, \mathrm{Em}=3\right)$

\begin{tabular}{|l|l|l|l|}
\hline $\mathrm{x}$ & $\mathrm{G}_{\mathrm{m}}=1$ & $\mathrm{G}_{\mathrm{m}}=3$ & $\mathrm{G}_{\mathrm{m}}=5$ \\
\hline 0.0 & 7.30205 & 22.0037 & 36.70534 \\
\hline
\end{tabular}

\begin{tabular}{|l|l|l|l|}
\hline 0.4 & 8.54303 & 25.72656 & 42.910105 \\
\hline 0.8 & 1.18121 & 3.56543 & 5.94964 \\
\hline
\end{tabular}

TABLE -3

Variation of heat transfer with respect to $\sigma^{2}$ $\left(\mathrm{E}_{\mathrm{m}}=3, \mathrm{G}_{\mathrm{m}}=3, \varepsilon=0.1\right)$

\begin{tabular}{|l|l|l|l|}
\hline $\mathrm{x}$ & $\sigma^{2}=1$ & $\sigma^{2}=2$ & $\sigma^{2}=3$ \\
\hline 0.0 & 22.42945 & 22.0037 & 21.99219 \\
\hline 0.4 & 25.94013 & 25.72656 & 25.451301 \\
\hline 0.8 & 3.76532 & 3.56543 & 3.47135 \\
\hline
\end{tabular}

TABLE -4

Heat transfer variation with $\varepsilon$

$$
\left(\mathrm{E}_{\mathrm{m}}=3, \mathrm{G}_{\mathrm{m}}=3, \sigma^{2}=2\right)
$$

\begin{tabular}{|l|l|l|l|}
\hline $\mathrm{x}$ & $\varepsilon=0$ & $\varepsilon=0.1$ & $\varepsilon=0.2$ \\
\hline 0.0 & 0.0 & 22.0037 & 44.0073 \\
\hline 0.4 & 0.0 & 25.72656 & 72.89057 \\
\hline 0.8 & 0.0 & 3.56543 & 3.763 \\
\hline
\end{tabular}

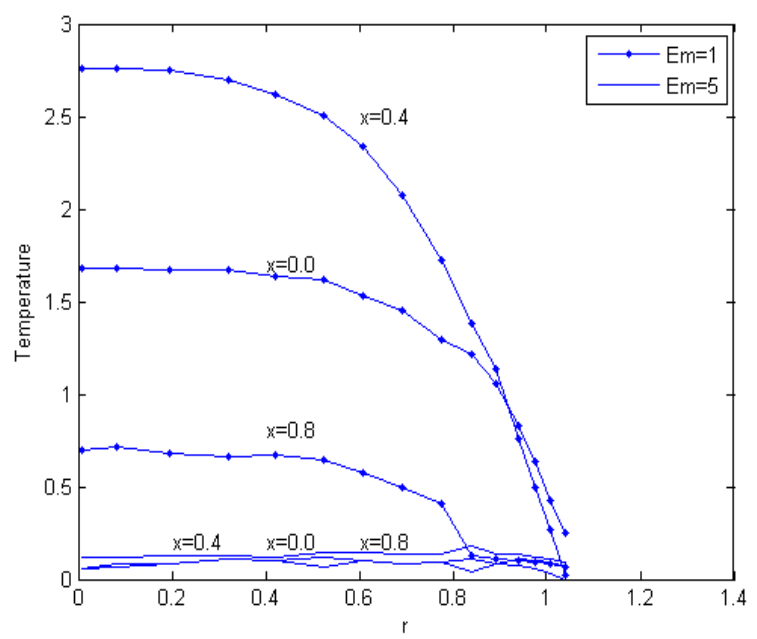

Fig-1, Temperature variation with $E m$ for the fixed values of $G_{m}=3, \sigma^{2}=2$ and $\varepsilon=0.1$

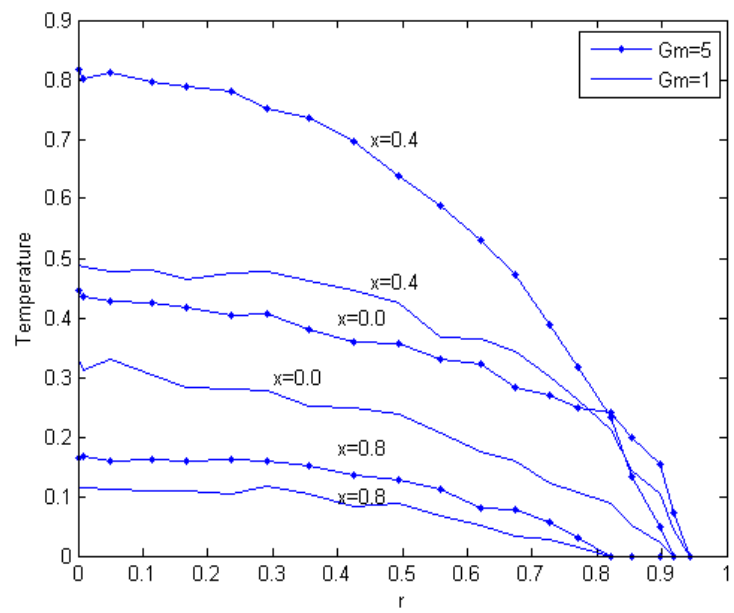

Fig-2, Temperature variation with $G m$ for the fixed values of $\varepsilon=0.1, \sigma^{2}=2$ and $E_{m}=3$ 


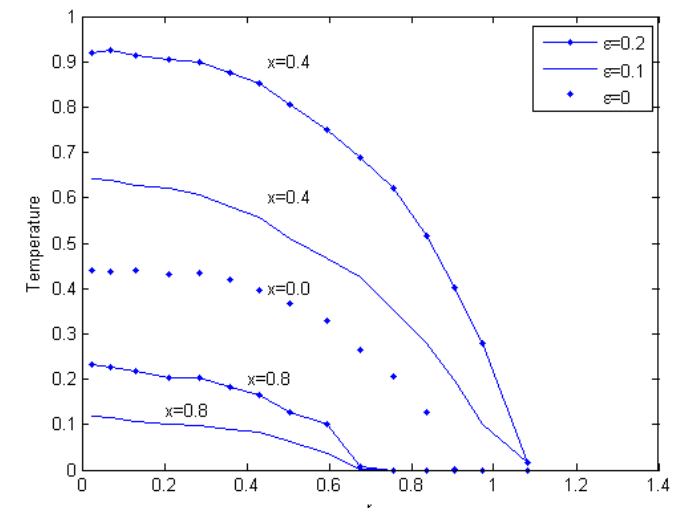

Fig-3, Temperature variation with $\varepsilon$ for the fixed values of $G_{m}=3, \sigma^{2}=2$ and $E_{m}=3$

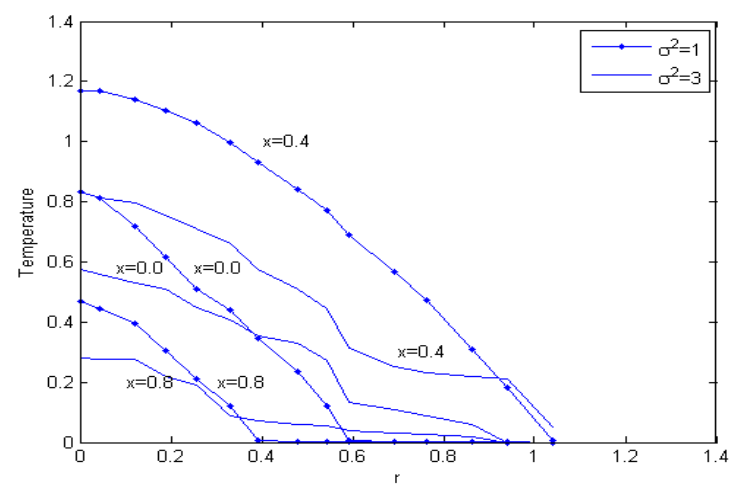

Fig-4, Temperature variation with $\sigma^{2}$ for the fixed

$$
\text { values of } \begin{aligned}
G_{m}= & 3, \varepsilon=0.1 \text { and } E_{m}=3 \\
& @ @ @ @
\end{aligned}
$$

\section{REFERENCES}

[1] G.Radhakrishnamacharya, Long Wave Length Approximation To Peristaltic Motion Of Power-Law Fluid, Rheol.Acta, 21(1982), 30-35.

[2] S.Takabatake, K.Ayukawa And A.MORI, Peristaltic Pumping In Circular Cylindrical Tubes: A Numerical Study Of Fluid Transport And Its Efficiency, J. Fluid Mech., 193(1988), 267-283.

[3] G.RadhakrishnamacharyaAnd V.Radhakrishna Murthy, Heat Transfer To Peristaltic Transport In A Non-Uniform Channel, Def. Sci. J., 43(3)(1993), 275-280.

[4] J.B.Shukla, R.S.Parihar, B.H.P.Rao And S.P.Gupta, Effect Of Peripheral Layer Viscosity On Peristaltic Transport Of Bio-Fluid, J.Fluid Mech., 97(1980), 225-237.

[5]. R Sivaiah And R Hemadri Reddy, Magnetohydrodynamic Peristaltic Motion Of A Newtonian Fluid Through Porous Walls Through Suction And Injection, IOP Conf. Series: Materials Science And Engineering 263 (2017) 062007.

[6] Singh, U. P.; Medhavi, Amit; Gupta, R. S.; Bhatt, Siddharth Shankar, Analysis Of Peristaltic Transport Of Non-Newtonian Fluids Through Nonuniform Tubes: Rabinowitsch Fluid Model, Zeitschrift Für Naturforschung A, Volume 72, Issue 7, Pp.601-608, 07/2017

[7] Nadeem, S., And Noreen Sher Akbar. "Influence Of Heat Transfer On Peristaltic Transport Of A Johnson-Segalman Fluid In An Inclined Asymmetric Channel." Communications In Nonlinear Science And Numerical Simulation 15.10 (2010): 2860-2877.

[8] Hayat, T., Najma Saleem, And N. Ali. "Effect Of Induced Magnetic Field On Peristaltic Transport Of A Carreau Fluid." Communications In Nonlinear Science And Numerical Simulation 15.9 (2010): 2407-2423.

[9] Vajravelu, K., Et Al. "Peristaltic Transport Of A Williamson Fluid In Asymmetric Channels With Permeable Walls." Nonlinear Analysis: Real World Applications 13.6 (2012): 2804-2822.

[10] Radhakrishnamacharya, G., And Ch Srinivasulu. "Influence Of Wall Properties On Peristaltic Transport With Heat Transfer." Comptes Rendus Mecanique 335.7 (2007): 369-373.

[11] Ellahi, R., M. Mubashir Bhatti, And K. Vafai. "Effects Of Heat And Mass Transfer On Peristaltic Flow In A Non-Uniform Rectangular
Duct." International Journal Of Heat And Mass Transfer 71 (2014): 706719.

[12] Bhatti, M. M., et al. "Mathematical modeling of heat and mass transfer effects on MHD peristaltic propulsion of two-phase flow through a Darcy-Brinkman-Forchheimer porous medium." Advanced Powder Technology 29.5 (2018): 1189-1197. 